\title{
"Fever of the South"
}

\author{
Cihan Cevik MD
}

"The infective endocarditis increases the physician's interest in the development of an infectious process".

William Bart Osler, 1893

\begin{abstract}
Medical historians report that Mozart suffered frequent attacks of tonsillitis, migratory polyarthritis, and glomerulonephritis and almost certainly had acute rheumatic fever. He underwent a dental extraction before penicillin was discovered. Subsequently, he developed fever and septic emboli and died at age 35 in 1791. The extent of infection in his heart still remains a mystery since this happened before the invention of ECGs, chest radiographs, and even the stethoscope. An autopsy was never done.
\end{abstract}

Approximately one hundred years later, William Osler was the first to describe infective endocarditis by reviewing the records of 200 patients with socalled ulcerative, malignant endocarditis in London. ${ }^{1}$ He included patients with fever and new murmurs, and he described his experience with a woman who developed erythematous, painful, swollen small spots occurring on the fingertips and feet resembling "beehives" that became known as Osler's nodes. In 1908, William Osler wrote that "with carefully made blood cultures one should now be able to determine the presence of the septicemia; this was easily done in three of my recent cases." 2

Infective endocarditis was almost $100 \%$ fatal until 1950s. Cure rates with sulfonamides $(5 \%) \mathrm{im}-$ proved up to $60-70 \%$ with the administration of penicillin. The concept of removing the infected tissue surgically from a patient with heart failure and fever seemed imprudent until Andrew Wallace excised and replaced the aortic valve with a Starr-Edwards prosthesis in a 45- year-old patient with Klebsiella endocarditis who was unresponsive to antibiotics. ${ }^{3}$ In the 21 st century, the risk of infective endocarditis remains a major concern, especially in patients with device implants, indwelling catheters, and IV drug abuse. These risk factors cause the tricuspid valve and the right heart to be involved before the left heart. Interestingly, the literature available on patients with rightsided infective endocarditis is much less than the literature available on mitral/aortic infective endocarditis cases, even though there is an increasing number of device implants and of IV drug users and decreasing prevalence of rheumatic heart valve disease.

Dr. Sutamtewagul and coworkers report the results of their chart review of the patients diagnosed with right-sided infective endocarditis between 2000 and 2011 in the current issue of the Journal. The authors speculated that embolic events associated with vegetations would increase pulmonary artery pressures, which, in turn, would predict hospital outcomes. The authors found out that most of these patients had mild pulmonary hypertension. Although they could not demonstrate an association between the mean pulmonary artery pressure and hospital outcomes, they observed that patients with right-sided infective endocarditis and abnormal chest radiographs stayed in the hospital three times longer than the patients with normal chest $x$-rays (7.5 vs. 21.4 days; $p=0.008$ ). Despite having the drawbacks of a retrospective, observational study using indirectly calculated pulmonary artery pressure data, their findings still underscore the utility of a simple chest $x$-ray for the triage, management, and perhaps outcome prediction in these patients. The lack of association between pulmonary artery pressure and length of stay seems to be a Type 2 error in this small study.

We are very fortunate for the advances in diagnosis and management of infective endocarditis 
in 21st century so that more than ninety percent of patients have favorable outcomes. While utilizing the best medical and surgical therapy, we should also keep in mind that simple diagnostic tools, such as a chest radiograph, may still help guide the management. Finally, we must not forget that an abnormality on the chest radiograph could also indicate other diseases, such as pneumonia, lung abscess, or empyema, conditions which caused Sir William Osler's death in 1916.

Corresponding Author: Cihan Cevik MD

Author Contact Information: Email-ccevik@sleh.com Author Affiliation: Cihan Cevik is a cardiology fellow at Texas Heart Institute at St. Luke's Episcopal Hospital/Baylor College of Medicine, Houston, TX.

DOI: 10.12746/swrccc2014.0206.065

\section{REFERENCES}

1. Osler W. Gulstonian lectures, on malignant endocarditis. $\mathrm{Br}$ Med J 1885 (Mar 7); 1(1262): 467-470.

2. Contrepois A. Notes on the early history of infective endocarditis and the development of an experimental model. Clin Infect Dis 1995; 20:461-466.

3. Wallace AG, Young WG Jr, Osterhout S. Treatment of acute bacterial endocarditis by valve excision and replacement. Circulation $1965 ; 31: 450-453$. 